\title{
Identification of the Principal Proteoglycan-binding Site in LDL A Single-Point Mutation in apo-B100 Severely Affects Proteoglycan Interaction without Affecting LDL Receptor Binding
}

\author{
Jan Borén, ${ }^{*}$ Katherine Olin, $\|$ Isabelle Lee, ${ }^{\star}$ Alan Chait,,$\|$ Thomas N. Wight, ${ }^{\| \uparrow}$ and Thomas L. Innerarity ${ }^{\star \neq \S}$ \\ * Gladstone Institute of Cardiovascular Disease and ${ }^{\ddagger}$ Cardiovascular Research Institute, ${ }^{\S}$ Department of Pathology, University of \\ California, San Francisco, California 94141-9100; and Departments of "Medicine and ${ }^{\mathbb{T}}$ Pathology, University of Washington, Seattle, \\ Washington 98195-0001
}

\begin{abstract}
The subendothelial retention of LDLs through their interaction with proteoglycans has been proposed to be a key process in the pathogenesis of atherosclerosis. In vitro studies have identified eight clusters of basic amino acids in delipidated apo-B100, the protein moiety of LDL, that bind the negatively charged proteoglycans. To determine which of these sites is functional on the surface of LDL particles, we analyzed the proteoglycan-binding activity of recombinant human LDL isolated from transgenic mice. Substitution of neutral amino acids for the basic amino acids residues in site B (residues 3359-3369) abolished both the receptorbinding and the proteoglycan-binding activities of the recombinant LDL. Chemical modification of the remaining basic residues caused only a marginal further reduction in proteoglycan binding, indicating that site $B$ is the primary proteoglycan-binding site of LDL. Although site B was essential for normal receptor-binding and proteoglycan-binding activities, these activities could be separated in recombinant LDL containing single-point mutation. Recombinant LDL with a K3363E mutation, in which a glutamic acid had been inserted into the basic cluster RKR in site $B$, had normal receptor binding but interacted defectively with proteoglycans; in contrast, another mutant LDL, R3500Q, displayed defective receptor binding but interacted normally with proteoglycans. LDL with normal receptor-binding activity but with severely impaired proteoglycan binding will be a unique resource for analyzing the importance of LDLproteoglycan interaction in atherogenesis. If the subendothelial retention of LDL by proteoglycans is the initial event in early atherosclerosis, then LDL with defective proteoglycan binding may have little or no atherogenic potential. ( $J$. Clin. Invest. 1998. 101:2658-2664.) Key words: lipoproteins • apo-B $\cdot$ proteoglycans $\bullet$ atherosclerosis $\bullet$ transgenic mice
\end{abstract}

Address correspondence to Jan Borén, M.D., Ph.D., Wallenberg Laboratory for Cardiovascular Research, Sahlgrenska University Hospital, Göteborg University, S-413 45 Göteborg, Sweden. Phone: 46-31-602210; FAX: 46-31-823762; E-mail: jan.boren@wlab.wall.gu.se

Received for publication 14 November 1997 and accepted in revised form 1 April 1998.

J. Clin. Invest.

(C) The American Society for Clinical Investigation, Inc. 0021-9738/98/06/2658/07 \$2.00

Volume 101, Number 12, June 1998, 2658-2664

http://www.jci.org

\section{Introduction}

High levels of LDL are a major risk factor for coronary disease and are the source of most of the cholesterol that accumulates in the arterial wall (1). The subendothelial retention of LDL appears to be a key pathogenic process in atherosclerosis, and several lines of evidence suggest that intramural retention of atherogenic lipoproteins involves the extracellular matrix, chiefly proteoglycans (2-4).

Proteoglycans contain long carbohydrate side-chains of glycosaminoglycans, which are covalently attached to a core protein by a glycosidic linkage. The glycosaminoglycans consist of repeating disaccharide units, all bearing negatively charged groups, usually sulfate or carbohydrate groups. In vitro, LDL bind with high affinity to many proteoglycans found in the artery wall, including dermatan sulfate proteoglycans (for example, biglycan) and chondroitin sulfate proteoglycans (for example, versican), which are produced by smooth muscle cells in response to PDGF or TGFß $(5,6)$. The interaction between LDL and proteoglycans apparently involves clusters of basic amino acids in apo-B100, the protein moiety of LDL, that interact with the negatively charged glycosaminoglycan proteoglycans (7-10), or by bridging molecules, such as apo-E or lipoprotein lipase (3).

The isolation of large fragments of apo-B100 from different regions characterized by concentrations of positive clusters indicated that up to eight specific regions in apo-B100 bind glycosaminoglycans (8-10). Weisgraber and Rall (9) identified two fragments, residues 3134-3209 and 3356-3489, that bind to heparin with the highest affinity. Recently Camejo and coworkers confirmed this finding and proposed that residues 3147-3157 and 3359-3367 may act cooperatively in the association with proteoglycans $(2,11)$. However, because these studies were carried out with delipidated apo-B fragments in the presence of urea or with short synthetic apo-B peptides, it is not clear which of the binding sites are functionally expressed on the surface of LDL particles. Some or many of these postulated glycosaminoglycan-binding sites may not be functional when apo-B is associated with LDL. For example, apo-E has two heparin-binding sites, but only one binds to heparin when apo-E is complexed with phospholipid (12). This heparin-binding site coincides with the LDL receptor-binding site of apo-E (12).

Recently, we developed a procedure for site-directed mutagenesis of the large apo-B gene to study the functional domains of apo-B100 when physiologically incorporated into native lipoproteins (13). Using this approach, we generated a series of human-apo-B transgenic mice and analyzed recombinant lipoproteins isolated from these animals (14). The results from this study now allow a broad understanding of the receptor-binding site of human apo-B100 (residues 3359-3369) and explain how sequences and mutations located distal to the 
receptor-binding region can modulate the affinity of apo-B100 for the LDL receptor. Our success in identifying the receptorbinding domain of apo-B100 suggested that the same procedures could be used to define functional sites, such as the proteoglycan-binding sites, on apo-B100 on an LDL particle.

For these studies, we used human recombinant LDL isolated from four different types of human apo-B100 transgenic mice. The first line expressed recombinant control LDL. Its receptor-binding activity is almost identical to that of LDL isolated from human plasma (14). The second transgenic mouse line expressed recombinant LDL that had a single amino acid mutation, the substitution of glutamine for the normally occurring arginine 3500 in apo-B100 (R3500Q). Previously, we proved that this mutation is identical to the one that causes defective receptor binding in the genetic disorder, familial defective apo-B100. Although it is outside the receptor-binding site (site B), this mutation produces a conformational change that disrupts receptor binding (14). The third transgenic mouse line expressed recombinant LDL in which the basic amino acids in site B (residues 3359-3369) were converted to neutral amino acids. The arginine residues were converted to serines, and the lysine residues to alanines (RK3359-3369SA). These changes virtually abolish the receptor-binding activity of the recombinant LDL; this finding, along with other evidence, demonstrated that site B is the receptor-binding site of LDL (14). The fourth transgenic mouse line expressed human recombinant LDL in which the lysine at residue 3363 of apo-B100 was changed to glutamic acid (K3363E).

\section{Methods}

Generation of truncated P1 plasmids and isolation of DNA fragments for mutagenesis. The $95-\mathrm{kb}$ apo-B P1 plasmid p158 (15) was prepared and modified by RecA-assisted restriction endonuclease (RARE) ${ }^{1}$ cleavage as described (13). Oligomers EcoRI-35763 (5' gaaaactcccacagcaagctaatgattatctgaattcattcaattgggagagacaagtttcac $3^{\prime}$ ) and EcoRI41496 ( $5^{\prime}$ cacaagtgaaatatctggttaggatagaattctcccagttttcacaatgaaaacatc $3^{\prime}$ ) were used to make a 5.7-kb-deleted P1 plasmid. A 5.7-kb fragment was isolated from the apo-B100 "Leu-Leu" plasmid with RARE cleavage using oligomers EcoRI-35763 and EcoRI-41496 and cloned into the $\mathrm{pZErO}-1$ vector (Invitrogen, Carlsbad, CA). The apoB100 "Leu-Leu" plasmid was used to increase the yield of apo-B100, since it contains a CAA-to-CTA mutation in codon 2153 that effectively abolished the formation of apo-B48, which is formed by an editing mechanism present in mouse livers (16).

Site-directed mutagenesis of P1 DNA. The pZErO-5.7kb plasmid was subjected to site-directed mutagenesis with the Morph System (5 Prime $\rightarrow 3$ Prime, Inc., Boulder, CO) using oligonucleotide K3363E ( $5^{\prime}$ caagattgacaagagaaaggggattgaag $3^{\prime}$ ) to mutate the lysine at reside 3363 to glutamic acid. The resulting plasmids were subjected to RARE cleavage with oligomers EcoRI-35763 and EcoRI-41496, and the mutated 5.7-kb fragment was isolated. After RARE cleavage of the 5.7-kb-deleted P1 plasmid with oligonucleotide EcoRI del. 5.7-kb (5' ggaaaactcccacagcaagctaatgattatctgaattctcccagtttcacaatgaaaacatc $3^{\prime}$ ), the mutated 5.7-kb fragment was ligated into the linearized and dephosphorylated 5.7-kb-deleted P1 vector (13).

Human apo-B transgenic mice. The transgenic mice were generated with a P1 bacteriophage clone (p158) (15) that spanned the human apo-B gene in which mutations had been introduced by RARE

1. Abbreviations used in this paper: CHAPS, 3-[3-cholamidopropyldimethylammonio]-1-propane-sulfonate; RARE, RecA-assisted restriction endonuclease. cleavage $(13,14)$. The genetic background of each of the human apo-B transgenic lines was mixed $(\sim 50 \%$ strain $\mathrm{C} 57 \mathrm{BL} / 6$ and $\sim 50 \%$ strain $\mathrm{SJL})$. Transgenic mice were identified at the time of weaning (21 d) by screening mouse plasma for human apo-B100 Western dot-blot and Western analysis with the mAb 1D1 (17). Mice were housed in a pathogen-free barrier facility operating on a 12-h light/12-h dark cycle and were fed rodent chow containing $4.5 \%$ fat (Ralston Purina, St. Louis, MO).

Isolation of recombinant lipoproteins. Blood from mice fasted for $5 \mathrm{~h}$ was collected by cardiac puncture into tubes containing EDTA (final concentration, $1 \mathrm{mg} / \mathrm{ml}$ ), and the plasma was mixed with butylated hydroxytoluene (final concentration, $25 \mu \mathrm{M}$ ), PMSF (final concentration, $1 \mathrm{mM}$ ), and aprotinin (final concentration, $10 \mathrm{U} / \mathrm{ml}$ ). The LDL $(d=1.02-1.05 \mathrm{~g} / \mathrm{ml})$ were isolated by sequential ultracentrifugation with a Ti 70 rotor and dialyzed against $150 \mathrm{mM} \mathrm{NaCl}$ and $0.01 \%$ EDTA, $\mathrm{pH} 7.4$; the mouse apo-E and apo-B were removed by immunoaffinity chromatography as described (14). Lipoproteins used for receptor-binding experiments were isolated and assayed within 1 wk. Human plasma LDL, isolated from a single blood donor, were included as a control in each experiment.

Modification of recombinant $L D L$. To selectively modify arginines or lysines in apo-B100, recombinant LDL were incubated with acetic anhydride or cyclohexadione, respectively. Acetylation of LDL was carried out as described by Basu et al. (18). In short, with continuous stirring in an ice-water bath, $0.5 \mathrm{mg}$ recombinant LDL in $1.0 \mathrm{ml}$ $0.15 \mathrm{M} \mathrm{NaCl}$ and $0.01 \%$ EDTA was mixed with $1.5 \mu \mathrm{l}$ saturated sodium acetate solution every 15 min over $1 \mathrm{~h}$. Cyclohexandione modification of LDL was performed as by Mahley et al. (19). $0.5 \mathrm{mg}$ recombinant LDL in $1 \mathrm{ml}$ of $0.15 \mathrm{M} \mathrm{NaCl}$ and $0.01 \%$ EDTA was mixed with $2 \mathrm{ml}$ of $0.15 \mathrm{M}$ 1,2-cyclohexanedione in $0.2 \mathrm{M}$ sodium borate buffer ( $\mathrm{pH} \mathrm{8.1)}$ and incubated at $35^{\circ} \mathrm{C}$ for $2 \mathrm{~h}$. The sample was then dialyzed for $48 \mathrm{~h}$ against $0.15 \mathrm{M} \mathrm{NaCl}$ and $0.01 \%$ EDTA at $4^{\circ} \mathrm{C}$.

Cell culture and receptor-binding assays. Human fibroblasts were cultured in DME containing 10\% FBS. 7 d before each experiment, the fibroblasts were plated into 12 -well cell culture dishes (22-mm diam per well) at $\sim 12,000$ cells/well in the same medium. $2 \mathrm{~d}$ before each experiment, the cells were transferred to DME containing $10 \%$ human lipoprotein-deficient serum. Normal human $\left.{ }^{[25} \mathrm{I}\right]$-labeled LDL $(2 \mu \mathrm{g} / \mathrm{ml})$, along with increasing concentrations of unlabeled lipoproteins, was added to the cells in DME containing $25 \mathrm{mM}$ Hepes and $10 \%$ human lipoprotein-deficient serum. After a 3-h incubation at $4{ }^{\circ} \mathrm{C}$, the surface-bound radioactivity was determined. The amount of unlabeled lipoproteins needed to compete $50 \%$ with $\left.{ }^{[25} \mathrm{I}\right]$-labeled LDL was determined from an exponential decay curve-fitting model (20).

Biglycan and versican isolation. Biglycan and versican were prepared from cultured human arterial smooth muscle cells metabolically labeled with $\left[{ }^{35} \mathrm{~S}_{\mathrm{SO}_{4}}\right.$, as described by Chang et al. (21). Briefly, cell medium was concentrated on DEAE-Sephacel mini-columns equilibrated in $8 \mathrm{M}$ urea, $0.25 \mathrm{M} \mathrm{NaCl}$, and $0.5 \%$ 3-[3-cholamidopropyl-diethylammonio]-1-propane-sulfonate (CHAPS). The [ $\left.{ }^{35} \mathrm{~S}\right]$-labeled proteoglycans were eluted with $8 \mathrm{M}$ urea, $3 \mathrm{M} \mathrm{NaCl}$, and $0.5 \%$ CHAPS and applied to a Sepharose CL-2B column equilibrated in $8 \mathrm{M}$ urea and $0.5 \%$ CHAPS. Small aliquots of the resulting fractions were counted to provide a profile of the separated material. The fractions were then combined into four pools: pool 1, $K_{\mathrm{av}}=0-0.2$; pool 2, $K_{\text {av }}=0.2-0.4$; pool 3, $K_{\text {av }}=0.4-0.55$; and pool 4, $K_{\text {av }}=0.55-0.8$. Eluted material in each pool was concentrated on Centricon-50 spin columns and dialyzed into buffer A used for binding assays. The bulk of the $\left[{ }^{35} \mathrm{~S}_{\mathrm{SO}_{4}}\right.$ radioactivity was present in pools 1 and 3 . Western blot analyses showed that pool 1 contained versican and was negative for perlecan, biglycan, and decorin. Pool 3 contained only biglycan and no immunoreactivity for perlecan, versican, or decorin. Only very small amounts of decorin were found in pool 4.

Gel mobility shift assay. The interaction between LDL and biglycan or versican was investigated by a gel mobility shift assay (22). Before the assay, the $\left[{ }^{35} \mathrm{~S}\right]$ biglycan and $\left[{ }^{35} \mathrm{~S}\right]$ versican preparations were dialyzed extensively at $4^{\circ} \mathrm{C}$ against $10 \mathrm{mM}$ Hepes, $150 \mathrm{mM} \mathrm{NaCl}, 5$ 
$\mathrm{mM} \mathrm{CaCl}$, and $2 \mathrm{mM} \mathrm{MgCl}_{2}$ (pH 7.4, buffer A), and the protein concentrations were determined (Bio-Rad Protein assay; Bio-Rad Laboratories, Hercules, CA) with bovine $\gamma$-globulin as the standard. Increasing concentrations of LDL were incubated with $\sim 2,000 \mathrm{dpm}$ of $\left[{ }^{35} \mathrm{~S}\right]$ biglycan or $\left[{ }^{35} \mathrm{~S}\right]$ versican for $1 \mathrm{~h}$ at $37^{\circ} \mathrm{C}$ in a total volume of $20 \mu \mathrm{l}$ of buffer A. $3 \mu$ l of bromphenol blue/glycerol (1:1, vol/vol) was added to the samples, and $20 \mu \mathrm{l}$ was loaded into wells of $0.7 \%$ NuSieve (FMC BioProducts, Rockland, ME) agarose gels prepared on GelBond film (FMC BioProducts). Electrophoresis was run for $3 \mathrm{~h}$ at $60 \mathrm{~V}$ with recirculating buffer $\left(10 \mathrm{mM}\right.$ Hepes, $2 \mathrm{mM} \mathrm{CaCl}_{2}, 4 \mathrm{mM}$ $\mathrm{MgCl}_{2}, \mathrm{pH} 7.2$ ) in a cold room. Gels were fixed with $0.1 \%$ cetyl pyridium chloride in $70 \%$ ethanol for $90 \mathrm{~min}$, air-dried, and exposed to Hyper Film-MP (Amersham Life Sciences, Arlington Heights, IL) at $-70^{\circ} \mathrm{C}$. The $\left[{ }^{35} \mathrm{~S}\right]$ biglycan or $\left[{ }^{35} \mathrm{~S}\right]$ versican complexed to LDL appears as a band at the origin of the gel, and the free, unbound proteoglycan migrates to the front. The autoradiographs were quantitatively evaluated with a Hewlett-Packard Scan Jet II cx and ImageQuant software (Molecular Dynamics, Sunnyvale, CA).

Analysis of the plasma lipoproteins. Purified lipoproteins were analyzed on Western blots of 3-15\% polyacrylamide-SDS gels with enhanced chemiluminescence Western blotting detection reagents (Amersham Life Sciences).

\section{Results}

Generation, isolation, and purification of recombinant human $L D L$. To identify the functional proteoglycan binding site(s) on LDL, we used RARE cleavage to mutate a human apoB100 P1 bacteriophage clone, generated transgenic mice expressing human recombinant LDL with defined mutations in apo-B100, and characterized the recombinant LDL.

LDL from four different lines of human apo-B100 transgenic lines were used (Table I). The first line contained human recombinant control apo-B100 (control apo-B100) without any mutation in the apo-B gene except a CAA-to-CTA mutation in codon 2153. This was done to improve the yield of recombinant LDL by eliminating the formation of apo-B48, which is formed by an editing mechanism present in mouse livers. The mutation does not interfere with the receptor-binding activity of the recombinant LDL (14). A second line of transgenic mice expressed human apo-B100 in which arginine at residue 3500 had been changed to glutamine (R3500Q). This mutation is located distal of the receptor-binding site, but causes the carboxyl-terminus of apo-B100 to disrupt the normal interaction between LDL and the LDL receptor (14). A third line of human-apo-B transgenic mice expressed apoB100 in which the arginine residues were converted to serines and the lysine residues were changed to alanines in the receptor-binding site of apo-B100 (RK3359-3369SA). The fourth line of human-apo-B transgenic mice expressed human apoB100 with lysine at residue 3363 mutated to glutamic acid (K3363E). This line was generated to investigate if the proteoglycan-binding and the receptor-binding activities can be separated.

Table I. Mutants of the Human Apo-B Gene

\begin{tabular}{lll}
\hline \multicolumn{1}{c}{ Recombinant LDL } & LDL receptor binding & Proteoglycan binding \\
\hline Control LDL & Normal & Normal \\
R3500Q LDL & Defective & Normal \\
RK3359-3369SA LDL & Defective & Defective \\
K3363E LDL & Normal & Defective \\
\hline
\end{tabular}

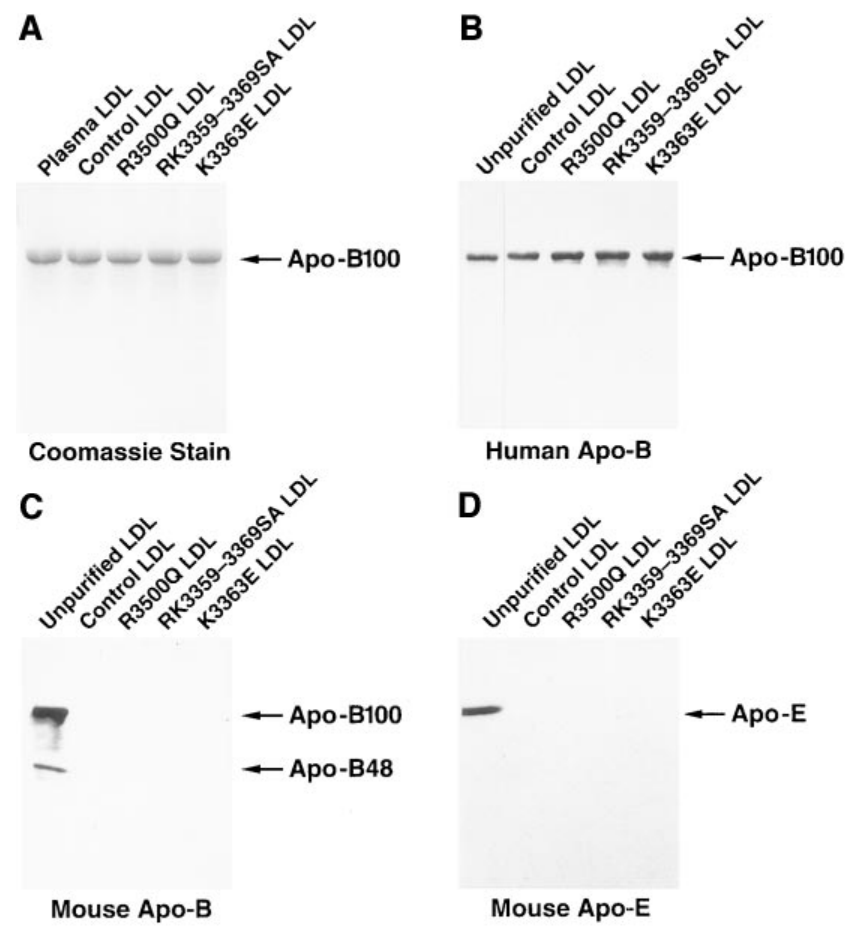

Figure 1. Coomassie staining and western analysis of recombinant LDL. Recombinant LDL $(d=1.02-1.05 \mathrm{~g} / \mathrm{ml})$ from four lines of human apo-B transgenic mice were isolated by sequential ultracentrifugation and subjected to immunoaffinity chromatography to remove endogenous apo-B and apo-E. $5 \mu \mathrm{g}$ of apo-B100 from human plasma LDL (lane 1) or immunopurified recombinant LDL (lanes 2-5) were analyzed by SDS-PAGE on 3-15\% gels $(A) .1 \mu \mathrm{g}$ each of unpurified recombinant control LDL (lane 1 ) and purified recombinant LDL (lanes 2-5) were analyzed by Western blots with mAb 1D1 against human apo-B $(B)$ and polyclonal antibodies against mouse apo-B $(C)$ or mouse apo-E $(D)$.

The recombinant LDL were isolated from the human-apo-B transgenic mouse plasma by ultracentrifugation and the endogenous mouse apo-E- and apo-B-containing lipoproteins were removed by immunoaffinity chromatography. Purified recombinant LDL isolated from the plasma of the four lines of transgenic mice contained intact apo-B100 without any visible contamination (Fig. $1 A$ ). Western blot analysis showed that the recombinant LDL from all four transgenic mouse lines bound to the mAb 1D1 (Fig. $1 B$ ), whose epitope is between amino acids 474 and 539 in human apo-B100 (17), and that only the unpurified recombinant LDL reacted with polyclonal antibodies against mouse apo-B and mouse apo-E (Fig. 1, $C$ and $D$, respectively), confirming that endogenous mouse apo-B and apo-E had been completely removed.

Competitive receptor-binding assays of mouse-derived human recombinant control LDL. To evaluate the receptorbinding activity of the recombinant LDL, LDL from each transgenic line were tested with an in vitro competitive receptor-binding assay (Fig. 2). Recombinant LDL with the uncharged site B (RK3359-3369SA) or with the R3500Q mutation had defective receptor binding $\left(\mathrm{ED}_{50}>20 \mu \mathrm{g} / \mathrm{ml}\right.$ for both), a finding in agreement with our previous observations (14). The K3363E LDL had normal receptor binding equivalent to that of control LDL (ED ${ }_{50} 2.4$ and $2.3 \mu \mathrm{g} / \mathrm{ml}$, respectively). 


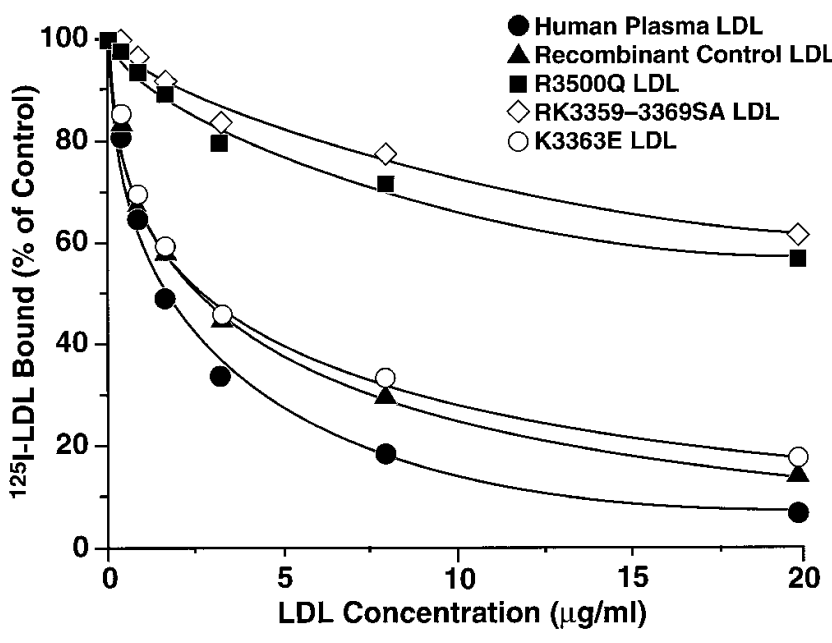

Figure 2. Competitive binding assay of recombinant LDL. The abilities of recombinant control LDL, R3500Q LDL, RK3359-3369SA LDL, and K3363E LDL to compete with [ $\left.{ }^{125} \mathrm{I}\right]$-labeled human plasma LDL $(2 \mu \mathrm{g} / \mathrm{ml})$ for binding to LDL receptors on normal human fibroblasts were determined. The recombinant lipoproteins were isolated from 15 mice, and endogenous apo-E and apo-B were removed. Competitor LDL were added at the indicated concentrations to normal human fibroblasts, and the amount of $\left[{ }^{125} \mathrm{I}\right] \mathrm{LDL}$ bound to the fibroblasts was measured after a 3 -h incubation. The results represent the average of data from three independent experiments performed with freshly isolated LDL for each experiment. Human plasma LDL were included as a control.

Gel shift analysis of recombinant $L D L$ with versican and biglycan. To determine the ability of the different recombinant LDL to interact with proteoglycans, the recombinant control, R3500Q, RK3359-3369SA, and K3363E LDL were isolated and subjected to gel-shift analysis (22). This procedure has the advantages that only microgram quantities of lipoproteins are required and the relative affinity of LDL binding to the proteoglycans can be determined at physiological ionic and $\mathrm{pH}$ conditions. In three independent experiments, recombinant control LDL and R3500Q LDL bound [ ${ }^{35}$ S] versican and $\left[{ }^{35} \mathrm{~S}\right]$ biglycan almost as efficiently as human plasma LDL, but recombinant RK3359-3369SA and K3363E LDL had severely impaired binding to both $\left[{ }^{35} \mathrm{~S}\right]$ versican and $\left[{ }^{35} \mathrm{~S}\right]$ biglycan (Fig. 3). Thus, mutations of basic amino acids in site B dramatically reduced the ability of apo-B100 to interact with proteoglycans. Of particular interest was that recombinant K3363E LDL interacted defectively with both versican and biglycan but had normal receptor binding. Recombinant control LDL with and without the "Leu-Leu" mutation displayed identical binding to $\left[{ }^{35} \mathrm{~S}\right]$ versican and $\left[{ }^{35} \mathrm{~S}\right]$ biglycan (data not shown). Thus, this mutation does not affect the binding of LDL to proteoglycans.

Gel shift analysis of acetylated or cyclohexanedione-modified recombinant $L D L$. Mutagenesis of site B severely reduced its interaction with versican and biglycan. To test the importance of the remaining clusters of basic amino acids for the interaction of LDL with versican or biglycan, the remaining arginines or lysines in apo-B100 were selectively modified to abolish the receptor-binding and heparin-binding activities of LDL (7). Recombinant LDL isolated from human apo-B transgenic mice expressing recombinant control or RK33593369SA LDL were divided into three aliquots. Two aliquots were selectively modified with acetic anhydride or cyclohexanedione to change the arginines and lysines, respectively. The ability of the modified recombinant control or RK33593369SA LDL to bind $\left[{ }^{35} \mathrm{~S}\right]$ biglycan or $\left[{ }^{35} \mathrm{~S}\right]$ versican was compared with that of unmodified recombinant LDL by gel shift analysis. Again, unmodified RK3359-3369SA LDL had greatly reduced ability to interact with $\left[{ }^{35} \mathrm{~S}\right]$ biglycan or $\left[{ }^{35}\right.$ S] versican (Fig. 4). Furthermore, the unmodified RK33593369SA LDL bound proteoglycans almost identically before and after chemical modification; only a minor difference was detected in the $\left[{ }^{35} \mathrm{~S}\right]$ biglycan gel shift assay (Fig. $4 \mathrm{~A}$ ). These data suggest that site $\mathrm{B}$ is the most important functional site for interaction with proteoglycans and that the seven other potential sites play a lesser role in apo-B100 (LDL).

\section{Discussion}

In this study, we examined the proteoglycan-binding activity of human recombinant LDL. Although eight glycosaminoglycanbinding sites have been identified in apo-B100 (8-10), it was
A

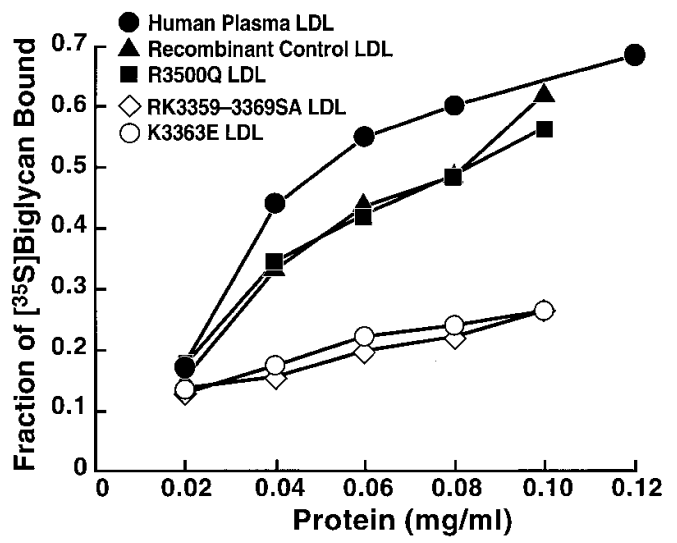

B

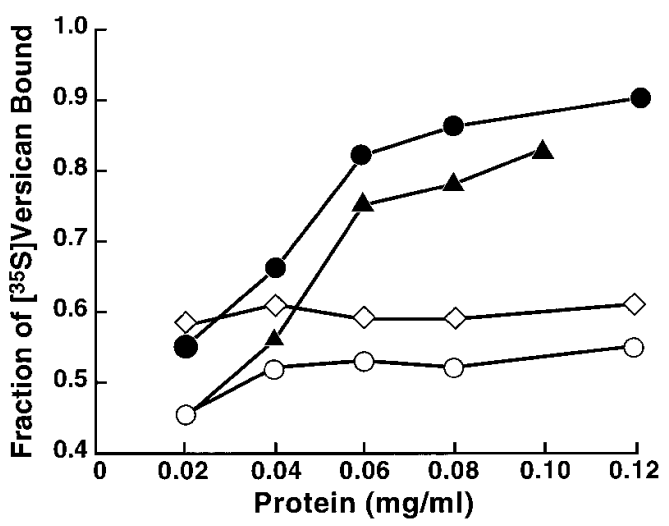

Figure 3. Gel shift analysis of mouse-derived recombinant LDL with $\left[{ }^{35}\right.$ S $]$ biglycan and $\left[{ }^{35} \mathrm{~S}\right]$ versican. The abilities of recombinant control LDL, R3500Q LDL, RK33593369SA LDL, and K3363E LDL to interact with $(A)$ $\left[{ }^{35}\right.$ S $]$ biglycan or $(B)\left[{ }^{35}\right.$ S $]$ versican were determined. The recombinant lipoproteins were isolated from 15 mice, and endogenous apo-E and apo-B were removed by immunoaffinity chromatography. The results represent the average data from three independent experiments

performed with freshly isolated LDL for each experiment. Human plasma LDL were included as a control. The high background in the gel shift analysis with $\left[{ }^{35} \mathrm{~S}\right]$ versican is nonspecific and due to precipitation of versican at the origin. 
A

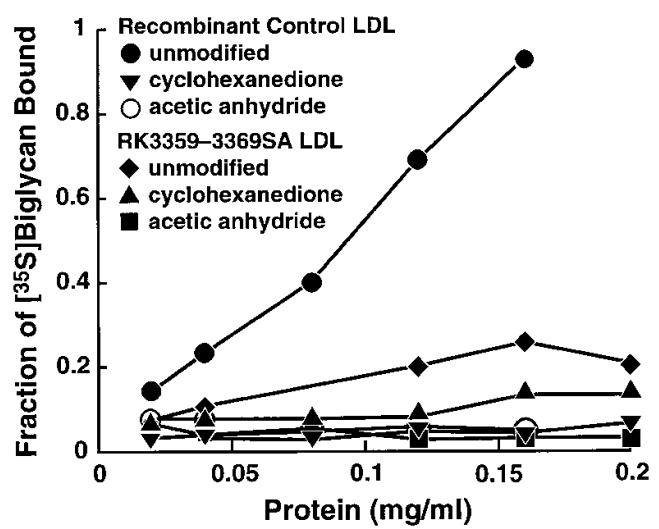

B

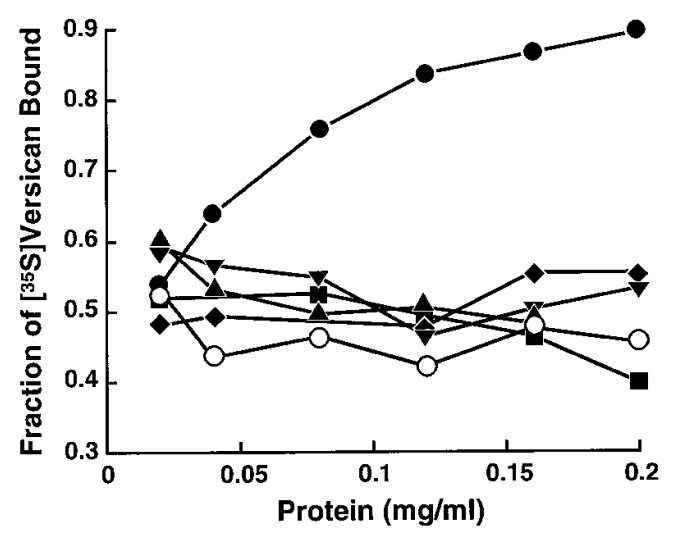

Figure 4. Gel shift analysis of mouse-derived recombinant LDL with ${ }^{35}$ S] versican and $\left[{ }^{35}\right.$ S $]$ biglycan after selective modification. The abilities of recombinant control LDL and RK3359-3369SA LDL, cyclohexanedione-modified control LDL and RK33593369SA LDL, and acetylated control LDL and RK3359-3369SA LDL to interact with $(A)\left[{ }^{35}\right.$ S]versican or $(B)\left[{ }^{35}\right.$ S $]$ biglycan were determined. The recombinant lipoproteins were isolated from 15 mice, and endogenous apo-E and apo-B were removed by immunoaffinity chromatography. The isolated recombinant LDL were treated with $(A)$ acetic anhydride or $(B)$ cyclohexanedione to selectively modify all arginines or lysines, respectively, in apo-B100. The high background in the gel shift analysis with $\left[{ }^{35} \mathrm{~S}\right]$ versican is nonspecific and due to precipitation of versican at the origin.

not known which of them participates in the physiological binding of LDL to proteoglycans. Previously, we identified site B (residues 3359-3369) as the LDL receptor-binding site (14), and in this study, we found that it is also the primary binding site for proteoglycans. We focused on site B because it is highly similar to the receptor-binding site of apo-E, which is also the physiological heparin-binding site (12) and because heparin competes with the LDL receptor for binding to LDL and displaces LDL from its receptor (23). Not only did studies on apo-E help us to focus on site $B$, but they also provided valuable clues for designing a single-point mutation in site $B$ that disrupts the proteoglycan binding but not the receptor binding of LDL. Genetically dominant forms of type III hyperlipidemia possess apo-E variants with single-point mutations in the receptor-binding site (24). Surprisingly, these mutations had much more severe effects on the binding of apo-E to heparin sulfate proteoglycans than to the LDL receptor (24). It is also known that acidic residues (aspartic or glutamic acid) inserted into clusters of basic residues are especially effective in disrupting the binding of proteins to proteoglycans (25). We thus reasoned that it might be possible to disrupt proteoglycan binding of site B by inserting a single glutamic acid in the center of the basic triad arginine3362-lysine3363-arginine3364 in site B. Recombinant LDL in which lysine 3363 in apo-B100 was changed to glutamic acid had severely defective proteoglycanbinding activity but had normal LDL receptor-binding activity. Thus, the proteoglycan-binding and the receptor-binding activities in LDL can be separated by introducing this singlepoint mutation into apo-B100. Moreover, since LDL with the K3363E mutation retained LDL receptor-binding activity, these results also indicate that the mutation did not affect the overall folding and stability of the protein. Furthermore, none of the mutations altered the size as determined by negativestaining EM or the lipid composition of the recombinant LDL (data not shown). As previously reported $(14,15,26)$ we found the human recombinant LDL was more triglyceride-rich than normal plasma-derived human LDL (data not shown).

The finding that a single amino acid substitution in the receptor-binding site of apo-B100 did not affect LDL receptor- binding activity may explain why, despite extensive searches, no mutations in the receptor-binding site (site B) have been found in patients with hypercholesterolemia (27). For example, Dunning et al. (28) examined the DNA of hypercholesterolemic patients with LDL that was defective in receptor binding and did not find any mutations in the gene encoding amino acids 3130-3630. Our study shows that a single-point mutation in the receptor-binding site, even one that disrupts the charge density, does not affect the receptor-binding activity and suggests that other single amino acid mutations in the B site may not greatly alter the receptor binding of LDL.

As discussed above, apo-B100 and apo-E share two properties that make them unique among plasma apolipoproteins: both bind to LDL receptors and both interact with a variety of glycosaminoglycans, including heparin. A comparison of these two functional domains has revealed an exceptional pattern of converging functional and structural evolution. Both apolipoproteins have a single receptor-binding site, and the two sites are strikingly similar. In fact, it is possible to swap them and retain full receptor-binding activity in the chimeric apolipoproteins (14). Both proteins have multiple heparin-binding sites when delipidated, but only one main site, which in both cases overlaps with the receptor-binding site, is functional when the proteins are incorporated into a lipid particle. Furthermore, in both proteins, single-point mutations that disrupt the charge of the receptor-binding sites affect the proteoglycan-binding activity more severely than the receptor-binding activity (24). Thus, binding of these apolipoproteins to the LDL receptor depends more on the correct conformation of the receptor-binding site, whereas binding to proteoglycans seems to be more dependent on clusters of positive charges in the receptor-binding site. This finding is in agreement with studies by Mahley and coworkers $(7,29)$ demonstrating that the interaction between lysine residues in apo-B100 and the LDL receptor are not simply an ionic interaction, since methylation of all lysine residues, which preserves the positive charge on the $\epsilon$-amino group, abolishes binding activity. In contrast, heparin binding to LDL is preserved when the positive charge on the lysine is maintained after reductive methylation but is prevented when 
the charge on the lysyl residues is abolished after neutralization of the positive charge by either acetoacetylation or carbamylation (7). Thus, although the same residues (arginine and lysine) in site B are involved in both receptor binding and proteoglycan binding of LDL, the nature of the chemical interaction appears to be different, and for receptor binding, the conformation of the site appears critical. For example, the mutations that most severely disrupt the receptor-binding activity in apo-B100 (R3500Q) and apo-E (R158C) are located distal to the receptor-binding sites. Both mutations cause a conformational change in and around the receptor-binding sites that virtually abolishes the receptor-binding activity, but has no or only a marginal effect on proteoglycan-binding activity (24).

Although our results indicate that site B is the primary site for the interaction of native LDL with proteoglycans, modification of the LDL may expose other proteoglycan-binding sites. Paananen and Kovanen (30) noted that proteolysis of apo-B100 strengthened the binding of LDL to proteoglycans, suggesting the exposure of buried heparin binding sites. Likewise, when LDL are fused by sphingomyelinase treatment, the modified lipoproteins bind more avidly to proteoglycans (30, 31). The finding that multiple heparin molecules bind to LDL (32) may also be explained by a cooperative effect of heparin binding to one site that triggers a conformational change in apo-B100 that enables other sites to participate in the interaction. Thus, the initial interaction with proteoglycans may induce structural alterations of the LDL that expose heparin/ proteoglycan-binding sites that may contribute to the intramural retention of LDL after the initial interaction with the primary binding site.

The ability to engineer a recombinant LDL with defective proteoglycan binding but normal receptor binding provides an opportunity to understand the potential importance of LDL binding to the artery wall proteoglycans in atherogenesis. The significance of this interaction has been highlighted in two recent review articles $(2,3)$. Williams and Tabas proposed that subendothelial retention of atherogenic lipoproteins is the central pathogenic process in atherosclerosis (3). Moreover, they hypothesized that retained lipoproteins can directly or indirectly provoke all known features of early lesions, such as lipoprotein oxidation, monocyte migration into the artery wall, macrophage foam cell formation, and cytokine production, and can accelerate further retention by stimulating local synthesis of proteoglycans. Several lines of evidence indicate that the retention of arterial lipoproteins involves the extracellular matrix; proteoglycans, in particular, appear to play an important role $(2,3,8,33)$. First, purified arterial proteoglycans, especially those from lesion-prone sites $(34,35)$, bind atherogenic lipoproteins in vitro, particularly LDL from patients with coronary artery disease (36). LDL binds with high affinity to dermatan sulfate and chondroitin sulfate, proteoglycans produced by proliferating smooth muscle cells (22). Second, proteoglycans are a major component of the artery wall extracellular matrix and are available to participate in the interactions of lipoproteins in the earliest stages of atherogenesis $(37,38)$. Third, retained apo-B immunologically co-localizes with proteoglycans in early and developed lesions (39-43). The observation that the arterial wall content of these proteoglycans increases during atherosclerosis and correlates with an increased accumulation of aortic cholesterol also supports the potential importance of the interaction between LDL and proteoglycans (41).
Our results indicate that site B (residues 3359-3369) is the major proteoglycan-binding site in apo-B100. These residues are only present on apo-B100, and not on apo-B48, which is truncated at residue 2153. However, gene-targeted mice expressing only apo-B48 developed, at similar high cholesterol levels, an equivalent degree of atherosclerosis as mice expressing only apo-B100 (44). Furthermore, the most widely used murine model for studying dislipidemia and atherosclerosis, obtained by inactivating the Apo-E gene $\left(\right.$ Apoe $\left.^{-/-}\right)$, contains mainly apo-B48-containing lipoproteins. The finding that mice with lipoproteins containing only or mainly apo-B48 develop severe atherosclerosis and our finding that the principal proteoglycan-binding site of LDL resides in the carboxyl-terminus of apo-B100 presents a paradox. If binding to artery wall proteoglycans is a key step in atherogenesis, then apo-B48-containing lipoproteins must bind proteoglycans via binding site(s) present in the amino-terminal $48 \%$ of the apo-B molecule that are masked or nonfunctional in apo-B100.

Our transgenic mice expressing recombinant human apoB100 LDL with the non-proteoglycan-binding mutations will give us a unique tool to investigate the importance of the LDL-proteoglycan interaction in the artery wall in the development of atherosclerosis. If the subendothelial retention of LDL by proteoglycans is the initial event in early atherosclerosis, then LDL with the K3363E mutation or other mutations that disrupt proteoglycan binding may have little or no atherogenic potential.

\section{Acknowledgments}

The authors thank Ms. K. Arnold for excellent help with receptorbinding assays; Dr. Susan Potter-Perigo for preparing the purified proteoglycans; Dr. Robert W. Mahley for stimulating discussions and support; Drs. Stanley C. Rall, Eva-Hurt Camejo, German Camejo and Karl H. Weisgraber for comments on the manuscript; S. Ordway and G. Howard for editorial assistance; and J. Carroll and S. Gonzales for graphics.

J. Borén was supported by a Howard Hughes Postdoctoral Fellowship for Physicians. This work was also supported by National Heart, Lung, and Blood Institute grants HL-47660, HL-18645, DK02456, and HL-30086.

\section{References}

1. Ross, R. 1995. Cell biology of atherosclerosis. Annu. Rev. Physiol. 57: 791-804.

2. Hurt-Camejo, E., U. Olsson, O. Wiklund, G. Bondjers, and G. Camejo. 1997. Cellular consequences of the association of apoB lipoproteins with proteoglycans. Potential contribution to atherogenesis. Arterioscler. Thromb. Vasc. Biol. 17:1011-1017.

3. Williams, K.J., and I. Tabas. 1995. The response-to-retention hypothesis of early atherogenesis. Arterioscler. Thromb. Vasc. Biol. 15:551-561.

4. Radhakrishnamurthy, B., S.R. Srinivasan, P. Vijayagopal, and G.S. Berenson. 1990. Arterial wall proteoglycans-biological properties related to pathogenesis of atherosclerosis. Eur. Heart J. 11 (Suppl. E):148-157.

5. Schönherr, E., H.T. Järveläinen, L.J. Sandell, and T.N. Wight. 1991. Effects of platelet-derived growth factor and transforming growth factor- $\beta 1$ on the synthesis of a large versican-like chondroitin sulfate proteoglycan by arterial smooth muscle cells. J. Biol. Chem. 266:17640-17647.

6. Schönherr, E., H.T. Järveläinen, M.G. Kinsella, L.J. Sandell, and T.N. Wight. 1993. Platelet-derived growth factor and transforming growth factor- $\beta_{1}$ differentially affect the synthesis of biglycan and decorin by monkey arterial smooth muscle cells. Arterioscler. Thromb. 13:1026-1036.

7. Mahley, R.W., K.H. Weisgraber, and T.L. Innerarity. 1979. Interaction of plasma lipoproteins containing apolipoproteins B and $\mathrm{E}$ with heparin and cell surface receptors. Biochim. Biophys. Acta. 575:81-91.

8. Camejo, G., S.-O. Olofsson, F. Lopez, P. Carlsson, and G. Bondjers. 1988. Identification of apo B-100 segments mediating the interaction of low density lipoproteins with arterial proteoglycans. Arteriosclerosis. 8:368-377. 
9. Weisgraber, K.H., and S.C. Rall, Jr. 1987. Human apolipoprotein B-100 heparin-binding sites. J. Biol. Chem. 262:11097-11103.

10. Hirose, N., D.T. Blankenship, M.A. Krivanek, R.L. Jackson, and A.D. Cardin. 1987. Isolation and characterization of four heparin-binding cyanogen bromide peptides of human plasma apolipoprotein B. Biochemistry. 26:55055512.

11. Olsson, U., G. Camejo, E. Hurt-Camejo, K. Elfsber, O. Wiklund, and G. Bondjers. 1997. Possible functional interactions of apolipoprotein B-100 segments that associate with cell proteoglycans and the apoB/E receptor. Arterioscler. Thromb. Vasc. Biol. 17:149-155.

12. Weisgraber, K.H., S.C. Rall, Jr., R.W. Mahley, R.W. Milne, Y.L. Marcel, and J.T. Sparrow. 1986. Human apolipoprotein E. Determination of the heparin binding sites of apolipoprotein E3. J. Biol. Chem. 261:2068-2076.

13. Borén, J., I. Lee, M.J. Callow, E.M. Rubin, and T.L. Innerarity. 1996. A simple and efficient method for making site-directed mutants, deletions, and fusions of large DNA such as P1 and BAC clones. Genome Res. 6:1123-1130.

14. Borén, J., I. Lee, W. Zhu, K. Arnold, S. Taylor, and T.L. Innerarity. Identification of the low density lipoprotein receptor-binding site in apolipoprotein B100 and the modulation of its binding activity by the carboxyl terminus in familial defective apo-B100. J. Clin. Invest. 101:1084-1093.

15. Linton, M.F., R.V. Farese, Jr., G. Chiesa, D.S. Grass, P. Chin, R.E. Hammer, H.H. Hobbs, and S.G. Young. 1993. Transgenic mice expressing high plasma concentrations of human apolipoprotein B100 and lipoprotein(a). $J$. Clin. Invest. 92:3029-3037.

16. Yao, Z., B.D. Blackhart, D.F. Johnson, S.M. Taylor, K.W. Haubold, and B.J. McCarthy. 1992. Elimination of apolipoprotein B48 formation in rat hepatoma cell lines transfected with mutant human apolipoprotein B cDNA constructs. J. Biol. Chem. 267:1175-1182.

17. Milne, R.W., R. Theolis, Jr., R.B. Verdery, and Y.L. Marcel. 1983. Characterization of monoclonal antibodies against human low density lipoprotein. Arteriosclerosis. 3.23-30.

18. Basu, S.K., J.L. Goldstein, R.G.W. Anderson, and M.S. Brown. 1976. Degradation of cationized low density lipoprotein and regulation of cholesterol metabolism in homozygous familial hypercholesterolemia fibroblasts. Proc. Natl. Acad. Sci. USA. 73:3178-3182.

19. Mahley, R.W., T.L. Innerarity, R.E. Pitas, K.H. Weisgraber, J.H. Brown, and E. Gross. 1977. Inhibition of lipoprotein binding to cell surface receptors of fibroblasts following selective modification of arginyl residues in arginine-rich and B apoproteins. J. Biol. Chem. 252:7279-7287.

20. Arnold, K.S., T.L. Innerarity, R.E. Pitas, and R.W. Mahley. 1992. Lipoprotein-receptor interactions. In Lipoprotein Analysis. A Practical Approach. C.A. Converse and E.R. Skinner, editors. Oxford University Press, Oxford. $145-168$

21. Chang, Y., M. Yanagishita, V.C. Hascall, and T.N. Wight. 1983. Proteoglycans synthesized by smooth muscle cells derived from monkey (Macaca nemestrina) aorta. J. Biol. Chem. 258:5679-5688.

22. Camejo, G., G. Fager, B. Rosengren, E. Hurt-Camejo, and G. Bondjers. 1993. Binding of low density lipoproteins by proteoglycans synthesized by proliferating and quiescent human arterial smooth muscle cells. J. Biol. Chem. 268: 14131-14137.

23. Goldstein, J.L., S.K. Basu, G.Y. Brunschede, and M.S. Brown. 1976. Release of low density lipoprotein from its cell surface receptor by sulfated glycosaminoglycans. Cell. 7:85-95.

24. Ji, Z.-S., S. Fazio, and R.W. Mahley. 1994. Variable heparan sulfate proteoglycan binding of apolipoprotein E variants may modulate the expression of type III hyperlipoproteinemia. J. Biol. Chem. 269:13421-13428.

25. Jackson, R.L., S.J. Busch, and A.D. Cardin. 1991. Glycosaminoglycans: Molecular properties, protein interactions, and role in physiological processes. Physiol. Rev. 71:481-539.

26. McCormick, S.P.A., J.K. Ng, M. Véniant, J. Borén, V. Pierotti, L.M. Flynn, D.S. Grass, A. Connolly, and S.G. Young. 1996. Transgenic mice that overexpress mouse apolipoprotein B. Evidence that the DNA sequences controlling intestinal expression of the apolipoprotein B gene are distant from the structural gene. J. Biol. Chem. 271:11963-11970.

27. Ludwig, E.H., P.N. Hopkins, A. Allen, L.L. Wu, R.R. Williams, J.L. Anderson, R.H. Ward, J.-M. Lalouel, and T.L. Innerarity. 1997. Association of genetic variations in apolipoprotein B with hypercholesterolemia, coronary artery disease, and receptor binding of low density lipoproteins. J. Lipid Res. 38 $1361-1373$.

28. Dunning, A.M., R. Houlston, J. Frostegård, J. Revill, J. Nilsson, A. Hamsten, P. Talmud, and S. Humphries. 1991. Genetic evidence that the putative receptor binding domain of apolipoprotein B (residues 3130 to 3630 ) is not the only region of the protein involved in interaction with the low density lipoprotein receptor. Biochim. Biophys. Acta. 1096:231-237.

29. Weisgraber, K.H., T.L. Innerarity, and R.W. Mahley. 1978. Role of the lysine residues of plasma lipoproteins in high affinity binding to cell surface receptors on human fibroblasts. J. Biol. Chem. 253:9053-9062.

30. Paananen, K., and P.T. Kovanen. 1994. Proteolysis and fusion of low density lipoprotein particles independently strengthen their binding to exocytosed mast cell granules. J. Biol. Chem. 269:2023-2031.

31. Tabas, I., Y. Li, R.W. Brocia, S.W. Xu, T.L. Swenson, and K.J. Williams. 1993. Lipoprotein lipase and sphingomyelinase synergistically enhance the association of atherogenic lipoproteins with smooth muscle cells and extracellular matrix. A possible mechanism for low density lipoprotein and lipoprotein(a) retention and macrophage foam cell formation. J. Biol. Chem. 268:20419-20432.

32. Cardin, A.D., C.J. Randall, N. Hirose, and R.L. Jackson. 1987. Physicalchemical interaction of heparin and human plasma low-density lipoproteins. Biochemistry. 26:5513-5518.

33. Hurt, E., and G. Camejo. 1987. Effect of arterial proteoglycans on the interaction of LDL with human monocyte-derived macrophages. Atherosclerosis. 67:115-126.

34. Cardoso, L.E.M., and P.A.S. Mourão. 1994. Glycosaminoglycan frac tions from human arteries presenting diverse susceptibilities to atherosclerosis have different binding affinities to plasma LDL. Arterioscler. Thromb. 14:115124

35. Ismail, N.A.E., M.Z. Alavi, and S. Moore. 1994. Lipoprotein-proteoglycan complexes from injured rabbit aortas accelerate lipoprotein uptake by arterial smooth muscle cells. Atherosclerosis. 105:79-87.

36. Lindén, T., G. Bondjers, G. Camejo, R. Bergstrand, L. Wilhelmsen, and O. Wiklund. 1989. Affinity of LDL to a human arterial proteoglycan among male survivors of myocardial infarction. Eur. J. Clin. Invest. 19:38-44.

37. Camejo, G., E. Hurt-Camejo, U. Olsson, and G. Bondjers. 1993. Proteoglycans and lipoproteins in atherosclerosis. Curr. Op. Lipidol. 4:385-391.

38. Camejo, G., B. Rosengren, U. Olson, F. Lopez, S.-O. Olofson, C. Westerlund, and G. Bondjers. 1990. Molecular basis of the association of arterial proteoglycans with low density lipoproteins: Its effect on the structure of the lipoprotein particle. Eur. Heart J. 11 (Suppl. E):164-173.

39. Walton, K.W., and N. Williamson. 1968. Histological and immunofluorescent studies on the evolution of the human atheromatous plaque. J. Atheroscler. Res. 8:599-624.

40. Hoff, H.F., and M.G. Bond. 1983. Apolipoprotein B localization in coronary atherosclerotic plaques from cynomolgus monkeys. Artery. 12:104-116.

41. Hoff, H.F., and W.D. Wagner. 1986. Plasma low density lipoprotein accumulation in aortas of hypercholesterolemic swine correlates with modifications in aortic glycosaminoglycan composition. Atherosclerosis. 61:231-236.

42. Nievelstein-Post, P., G. Mottino, A. Fogelman, and J. Frank. 1994. An ultrastructural study of lipoprotein accumulation in cardiac valves of the rabbit. Arterioscler. Thromb. 14:1151-1161.

43. Galis, Z.S., M.Z. Alavi, and S. Moore. 1993. Co-localization of aortic apolipoprotein B and chondroitin sulfate in an injury model of atherosclerosis Am. J. Pathol. 142:1432-1438.

44. Véniant, M.M., V. Pierotti, D. Newland, C.M. Cham, D.A. Sanan, R.L. Walzem, and S.G. Young. 1997. Susceptibility to atherosclerosis in mice expressing exclusively apolipoprotein B48 or apolipoprotein B100. J. Clin. Invest. 100:180-188. 